\title{
Nianguiry Kanté (circa 1951-2021)
}

e fut à Maastricht, en 1998, une réunion de dénommés “ experts " de la CEE autour de la question de la santé mentale dans les pays en voie de développements - on parlerait aujourd'hui des infortunes de la folie dans les pays extra européens mais c'est presque une autre histoire aujourd'hui. Invité par l'anthropologue et psychanalyste Jaak le Roy, grand amoureux et connaisseur de l'Afrique Centrale, j'y rencontrai Nianguiry pour la première fois. L'ami Kanté, avec ce qu'il faut de distance rieuse et avisée, sut se rendre infiniment présent dans tous les débats il a tout de suite gagné mon estime. Il avait pour le Mali et la santé publique des projets, ce qui est bien, des convictions, ce qui est nécessaire, et un sens éminent de l'alliance des projets et des convictions ce qui autant indispensable que rare.

Allions-nous nous perdre de vue ? Je le redoutais, il n’en fut rien. Expédié en mission à Bamako par mon indéfectible ami, Xavier Emmanuelli et pour le compte du Samu Social international, j'allais à Bamako définir puis mettre en place un dispositif d'accueil d'urgence des mineurs les plus en périls dans la vie en rue. Avec le professeur Baba Koumaré, Nianguiry Kanté, ingénieur de recherche à l'Institut de la Santé Publique fut un de mes interlocuteurs les plus disponibles et les plus fiables. Les prémices de notre douce et constante amitié s'en virent alors renforcés. Nous étions dans la joie résolue d'un accord sur les principes de l'action sociale en faveur des plus démunis et des opprimés. La question sanitaire insiste; les personnes âgés, les enfants errants, et les femmes en rupture de ban sont les plus vulnérables, Vers ces personnes s'il faut aller, il faut d'abord les écouter pour agir en raison.

Des convergences de vue existaient et se dessinaient des synergies d'action. Dans le creuset de l'exigence scientifique, dans le souci de la justice sanitaire, et avivés par le doux aiguillon de l'amitié, un projet hasardeux, comme la plupart des projets promis à un joyeux développement, vit le jour. Celui de fonder et de faire fonctionner une revue africaine centrée sur la question de la santé dans ses aspects sociaux, collectifs, culturels, dans ses liens avec les irruptions de la modernité, dans sa consistance clinique aussi.

Nianguiry Kanté, que vite rejoignirent ses amis et partenaires maliens, et moi-même, jetions alors les bases de la Revue africains des sciences sociales et de la santé publique dont je suis toujours co-rédacteur en chef. Cette revue est promise à un long avenir, connue dans tout le bassin de l'Afrique de l'Ouest et au-delà, elle est le fait de tisseurs de liens, généreux et intelligents.

Le Professeur Kanté termina une brillante carrière en tant que Directeur de recherche (spécialité sociologie) de classe exceptionnelle. Il dirigea plusieurs dizaines de thèses (une soixantaine) dont récemment celle de Fanta Sekou Sow intitulée: 
“Les déterminants de la déperdition scolaire dans l'enseignement fondamental au Mali : cas des écoles fondamentales du Centres d'Animation Pédagogique de BacoDjicoroni, Académie d'Enseignement Rive Droite du District de Bamako ", celle de M. Modibo Keita intitulée : “Perception et prise en charge de la malnutrition chez les enfants de 6 à 59 mois au Mali. Cas des régions de Kayes, Sikasso et du District de Bamako. ", et celle de M. Amadou Diabate intitulée : "Recours aux soins pédiatriques traditionnels dans la commune Urbaine de Ségou et communes rurales de Péléngana et de Sakoiba (Ségou, Mali)». Toutes les thèses dirigées par N. Kanté furent saluées et reconnues comme dignes de la plus haute mention par leur jury respectif.

Il fut, depuis 2012-membre fondateur et trésorier adjoint du congrès Panafricain des Anthropologues. Collaborateur externe du département d'anthropologie de l'université de Paris VIII, Saint Denis, France ; collaborateur externe du laboratoire " centre de recherches psychanalyse, médecine et sociétés " (EA 3522) de l'université Paris 7, Paris, France ;nous le connaissions aussi en tant que correspondant à Bamako (Mali) de notre revue Psychologie Clinique, de même était-il membre du Comité scientifique de Revue Sénégalaise de la Sociologie.

Je ne peux terminer sans rendre hommage à sa générosité, sa probité, son envie féconde que les hommes de bonne volonté et de bon sens puissent faire ouvre ensemble. Nianguiry a réussi ce défi dans une dynamique pragmatique et éthique nécessaire.

Un mot enfin, davantage personnel, Nianguiry Kanté fut un homme intègre, clairvoyant et fidèle. Il pouvait toujours compter sur moi et ce fut bien réciproque. Je salue sa famille avec reconnaissance; j'étais heureux de rencontrer son épouse et ses sept enfants, dans le doux climat de la confiance et de l'amitié. Notre peine est immense.

Olivier Douville Corédacteur en chef de la Revue Africaine des Sciences Sociales et de la Santé Paris, ce 13 septembre 2021 